\title{
HIV prevalence among clients attending a sexually transmitted diseases clinic in Amsterdam: the potential risk for heterosexual transmission
}

\author{
J S A Fennema, E J C van Ameijden, R A Coutinho, G J J van Doornum, \\ C J M Henquet, J A R van den Hoek
}

\begin{abstract}
Introduction-Patients attending a clinic for sexually transmitted diseases (STD) in general have engaged in at risk sexual behaviour. Therefore they are at increased risk of acquiring HIV through sexual contact.
\end{abstract}

Objective-To determine the HIV prevalence among patients attending a STD clinic in Amsterdam.

Methods-An anonymous cross sectional study was conducted in two 5-week periods in Spring and Autumn 1991.

Results -Of the 2362 patients attending the clinic during the study period, 2292 (97\%) consented to participate; of these, $2138(93 \%)$ were interviewed and anonymously tested, while $154(7 \%)$ consented to be interviewed but refused HIV antibody testing. The HIV prevalence was $4.2 \%(90 / 2138) ; 93 \%$ of seropositive participants reported homosexual contacts and/or intravenous use of drugs (IVDU). HIV prevalence among heterosexual non-IVDU men was $0.5 \%$ and among non-IVDU women $0.1 \%$. Among all heterosexually active participants, including IVDU and bisexual men, the HIV prevalence was $1.5 \%$. The 28 of 90 HIV infected participants that were heterosexually active reported together approximately 135 heterosexual partners in the six months preceding the study; 13 of these 28 heterosexually active participants had a STD diagnosed at their present clinic visit, while four $(30 \%)$ of them already knew they were HIV infected.

Conclusions-From these data we conclude that there is a substantial risk of further transmission of HIV through heterosexual contact. In order to try to reduce this potential for further sexual transmission of HIV, services offered by the STD clinic should not only include voluntary confidential counselling and HIV testing, but also notification of partners of HIV infected clinic-attendants. Finally, we conclude that anonymous HIV prevalence studies that link HIV test results to risk behaviour for HIV infection can be performed with a high rate of participation. Repeating such prevalence studies in time can help in monitoring the HIV incidence in the heterosexually active population.

(Genitourin Med 1993;69:23-28)

\section{Introduction}

The most common mode of transmission of the human immunodeficiency virus (HIV) is through sexual contact. ${ }^{1}$ Sexual transmission in Western European countries and the United States occurs predominantly among homosexual and bisexual men. The number of AIDS cases that are heterosexually acquired accounts for a small but slowly increasing proportion of the total number of reported cases. ${ }^{2}$ Of the 1950 cumulative AIDS cases reported in the Netherlands by 31 December $1991,6.5 \%$ is in the heterosexual transmission group. ${ }^{2}$

AIDS case surveillance data reflect, however, patterns of transmission of HIV that took place in the past and therefore do not provide information on the extent to which HIV has penetrated into the heterosexually active population. To obtain more recent information on trends in transmission patterns, sentinel surveys among populations at increased risk for HIV infection are important supplements to AIDS case surveillance. ${ }^{3}$ Patients attending sexually transmitted diseases (STD) clinics can serve as such a population; they include men who have had sex with men, intravenous drug users (IVDU) and heterosexual men and women who have engaged in sexual contact with multiple and/or anonymous partners.

In order to determine the HIV prevalence among STD clinic visitors, we conducted a voluntary, anonymous cross sectional study in which information on risk behaviour for HIV infection was linked to HIV test results and STD diagnoses. This study was conducted at the STD clinic operated by the Municipal Health Service of Amsterdam and forms part of a Concerted Action of the Medical and Health Research Council of the European Community, which aims at monitoring HIV infection rates over time in sentinel populations of STD patients in 12 European countries. 4

\section{Methods}

Study population

The STD clinic of the Municipal Health Service in Amsterdam offers anonymous and free of charge examination for STD. The clinic serves an important part of the city's population that has a relatively high risk of being infected with STD. ${ }^{5}$ Each visit made to the clinic for a possible new STD episode is considered a new consultation. Between 
12000 and 14000 new consultations are provided yearly to approximately $10000 \mathrm{clin}$ ic visitors. ${ }^{6}$ At each new consultation information on gender, age, nationality, place of residence, (IV) drug use, sexual orientation and STD diagnosis is routinely collected and entered into a computer under a patient identification code. All patients are routinely screened for gonorrhoea (GO), Chlamydia trachomatis $(\mathrm{CT})$ and syphilis.

\section{Data collection}

As part of a Concerted Action of the European Community, ${ }^{4}$ a cross sectional anonymous HIV prevalence study was performed in the clinic during two separate 5week periods in Spring and Autumn 1991.

Visitors who attended the clinic for a new consultation in this study period were asked to participate. Written information on the study and its anonymous character was available in six different languages. Informed consent was obtained of visitors who agreed to participate. Specially trained nurses and native tongue speaking interviewers (Spanish and Turkish) collected information on demographic characteristics, risk behaviour for HIV infection and previous HIV test results. A history of STD was defined as having had a prior episode of syphilis and/or gonorrhoea. Questions concerning sexual behaviour included homosexual contacts since 1980, sexual contact with HIV-positive partners ever, commercial sex-defined as sexual contact in exchange for money or drugs in the six months preceding the study-and the number of homosexual and heterosexual partners in the preceding 6 months, categorised as 1 , $2-4,5-10$ or $>10$ partners.

Participants were alse asked about a history of injecting drugs since 1980 and whether they ever received any transfusion with blood or blood products. When tested previously for HIV they were asked for the date of the last negative or the first positive test result.

Routine physical examination for STD was performed after completion of the questionnaire and drawing of a blood sample. A positive culture was used as diagnostic criterion for gonorrhoea; a diagnosis of CT infection was based on positive culture or ELISA. Primary herpes simplex infection was diagnosed by positive culture results in patients with ulcerations but without a history of prior genital herpes infection. A diagnosis of syphilis was based on clinical symptoms and a reactive serology (TPHA and FTA-absorbtion test) and/or demonstrating $T$ pallidum by dark field microscopy. ${ }^{6}$ Patients treated for syphilis in the past were diagnosed with a new syphilis infection only when a three fold or more increase in VDRL titre was observed or when $T$ pallidum was demonstrated. A diagnosis of trichomoniasis was based on direct microscopic examination of vaginal flora or, when negative, on culture. Laboratory-confirmed STD diagnoses were coded on the study-forms which then were separated from the patient's clinic-file and entered into a computer under its unique study number. This study was approved by the ethics committee of the Municipal Health Service of Amsterdam.

\section{Laboratory}

Blood was collected by venepuncture and tested for antibodies to HIV $1 / 2$ by means of recombinant HIV $1 / 2$ enzyme immunoassay (EIA) (Abbott GmbH, WiesbadenDelkenheim, Germany). Positive specimens were confirmed by Western Immunoblot (Diagnostic Biotechnology, Singapore).

Sera were screened for antibodies to hepatitis B virus (HBV) surface antigen (anti$\mathrm{HBs}, \mathrm{Ausab}$ ) and to core antigen (anti-HBc, Corzyme) using EIA (Abbott Laboratory, North Chicago, Ill., USA); testing for HBsAg (Auszyme) was performed only in specimens positive for anti-HBc and negative for antiHBs. Sera were considered reactive for $\mathrm{HBV}$ if at least anti-HBc or anti-HBs was positive; $\mathrm{HBV}$ carriers were defined as positive for $\mathrm{HBsAg}$ and anti-HBc but negative for antiHBs.

Treponema pallidum haemagglutination assay (TPHA, Fujirebio, Tokyo, Japan) was used for syphilis screening; when positive, the Venereal Disease Research Laboratory test (VDRL, Wellcome, Dartford, England) and the FTA-absorbtion test (Trepo-spot IF, Biomerieux, Marcy l'Etoile, France) were performed. All tests and cultures were performed at the Regional Laboratory of the Municipal Health Service of Amsterdam.

\section{Statistics}

The HIV prevalences in the various risk categories are given with $95 \%$ confidence intervals, using maximum likelihood techniques. Bivariate associations were analysed using chi square tests for categorical variables and by $t$ test for continuous variables. If expected cell frequencies were less than five, Fisher's exact test was used. P-values of $<0.05$ were considered as significant.

\section{Results}

\section{General description of participants}

Of the 2362 visitors who attended the clinic during the study period, 2292 (97\%) consented to participate. Of these, 2138 (93\%) were interviewed and tested for HIV antibodies, while $154(7 \%)$ consented to be interviewed but refused HIV antibody testing. Refusers were significantly more likely to originate from Surinam and the Dutch Antilles, to have a history of injecting drugs and to have a history of STD; refusers were less likely to be men who visited prostitutes. Between refusers and participants the proportion of those who already knew they were HIV seropositive did not differ (table 1). Knowledge of a previous HIV test result, either positive or negative, was reported by $255(12 \%)$ of the 2138 tested participants. 
Table 1 Demographic and other characteristics of 2292 participants who were tested and who refused testing for HIV

\begin{tabular}{|c|c|c|c|c|}
\hline Variable & $\begin{array}{l}\text { Tested } \\
n=2138 \\
n(\%)\end{array}$ & $\begin{array}{l}\text { Not tested } \\
n=154 \\
n(\%)\end{array}$ & $\begin{array}{l}\text { Total } \\
n=2292 \\
n(\%)\end{array}$ & \multirow{2}{*}{$\begin{array}{l}p \text { value } \\
0.64\end{array}$} \\
\hline Mean (sd) age (years) & $30.6 \quad(9 \cdot 0)$ & $30 \cdot 9 \quad(7 \cdot 8)$ & $30.7 \quad(8.9)$ & \\
\hline Gender-male & 1338 & $92(60)$ & $1430 \quad(62)$ & 0.48 \\
\hline Dutch nationality & $1431 \quad(67)$ & $114 \quad 74)$ & 1545 (67) & 0.053 \\
\hline Born in the Netherlands & $1117 \quad$ (52) & $74 \quad(48)$ & $1191 \quad(52)$ & 0.35 \\
\hline Ethnicity-Netherlands & $1124 \quad(53)$ & $76 \quad(50)$ & $1200 \quad(52)$ & \\
\hline -Surinam and Dutch Antilles & $222(10)$ & $33(21)$ & 255 (11) & \\
\hline -Turkey & $220 \quad(10)$ & 3 (2) & 223 (10) & \\
\hline -Southern Europe & $(1 \cdot 5)$ & (3) & (2) & 0.0002 \\
\hline - Northern Africa & (4) & (5) & (4) & \\
\hline -Other & $448 \quad(21)$ & (19) & $477 \quad(21)$ & \\
\hline Homosexual contact ever ${ }^{\star}$ & $324 \quad(24)$ & (29) & $351 \quad(25)$ & $0 \cdot 28$ \\
\hline History of IVDU ever* & (2) & (6) & (3) & 0.01 \\
\hline History of STD ever * & $650 \quad(31)$ & (42) & $715 \quad(32)$ & 0.004 \\
\hline Present STD diagnosis $\oint$ & $438 \quad(21)$ & $26 \quad(17)$ & $464 \quad(20)$ & 0.28 \\
\hline Visited prostitutes $\dagger$ & $440 \quad(33)$ & $21 \quad(23)$ & $461 \quad$ (33) & 0.045 \\
\hline Active as prostitute $\ddagger$ & $257 \quad(32)$ & 19 (32) & 275 (32) & 0.92 \\
\hline Known HIV seropositive & (2) & $(0 \cdot 6)$ & (2) & 0.20 \\
\hline
\end{tabular}

*since 1980

tsix months preceding the study, men only

tsix months preceding the study, women only

fincluding syphilis, gonorrhoea, genital chlamydia, primary genital herpes, first episodes of genital warts, trichomoniasis.

\section{HIV Prevalence}

Table 2 shows selected characteristics, STD diagnoses and the HIV prevalence by risk groups and gender of the 2138 participants who were tested for HIV.

The overall HIV prevalence was $4 \cdot 2 \%$ (90/2138; 95\%-CI:3·4-5.1\%). The HIV prevalence among homosexual men was $22 \%$ (70/324; 95\%-CI:18-27\%), among heterosexual male IVDUs $11 \%(2 / 17 ; 95 \%-$ CI:3-37\%) and among heterosexual men who never injected drugs $0.5 \% \quad(5 / 997$; 95\%-CI:0.2-1.2\%). The HIV prevalence among female IVDUs was $41 \%(12 / 29$; 95\%-CI:25-60\%) and among heterosexual women who never injected drugs the HIV prevalence was $0.1 \% \quad(1 / 771 ; 95 \%-$ CI:0.0-0.9\%). Six homosexual IVDUs, all HIV negative, were categorised as homo/ bisexual men.
The five men who are assumed to be HIV infected through heterosexual contact were all residents of Amsterdam. One was born in The Netherlands, two in Turkey, one in Pakistan and one in Ghana. None of these five men reported sexual contact with HIV infected partners or was a transfusion recipient. The one woman who is assumed to be heterosexually infected was Dutch and reported to have been tested negative for HIV in 1988 (table 3). Among heterosexually active participants-including bisexual men and IVDU-the HIV prevalence was $1.5 \%$ (28/1884; 95\%-CI:1·0-2.1\%).

Forty-eight of $90 \mathrm{HIV}$ infected patients knew the results of previous HIV tests; of these, 38 (79\%) already knew they were seropositive and 10 had tested negative previously (table 4). Taking into account these self reported previous HIV test results, six men

Table 2 General description and HIV prevalence among 2138 study participants ${ }^{*}$ who consented to HIV testing, by risk group

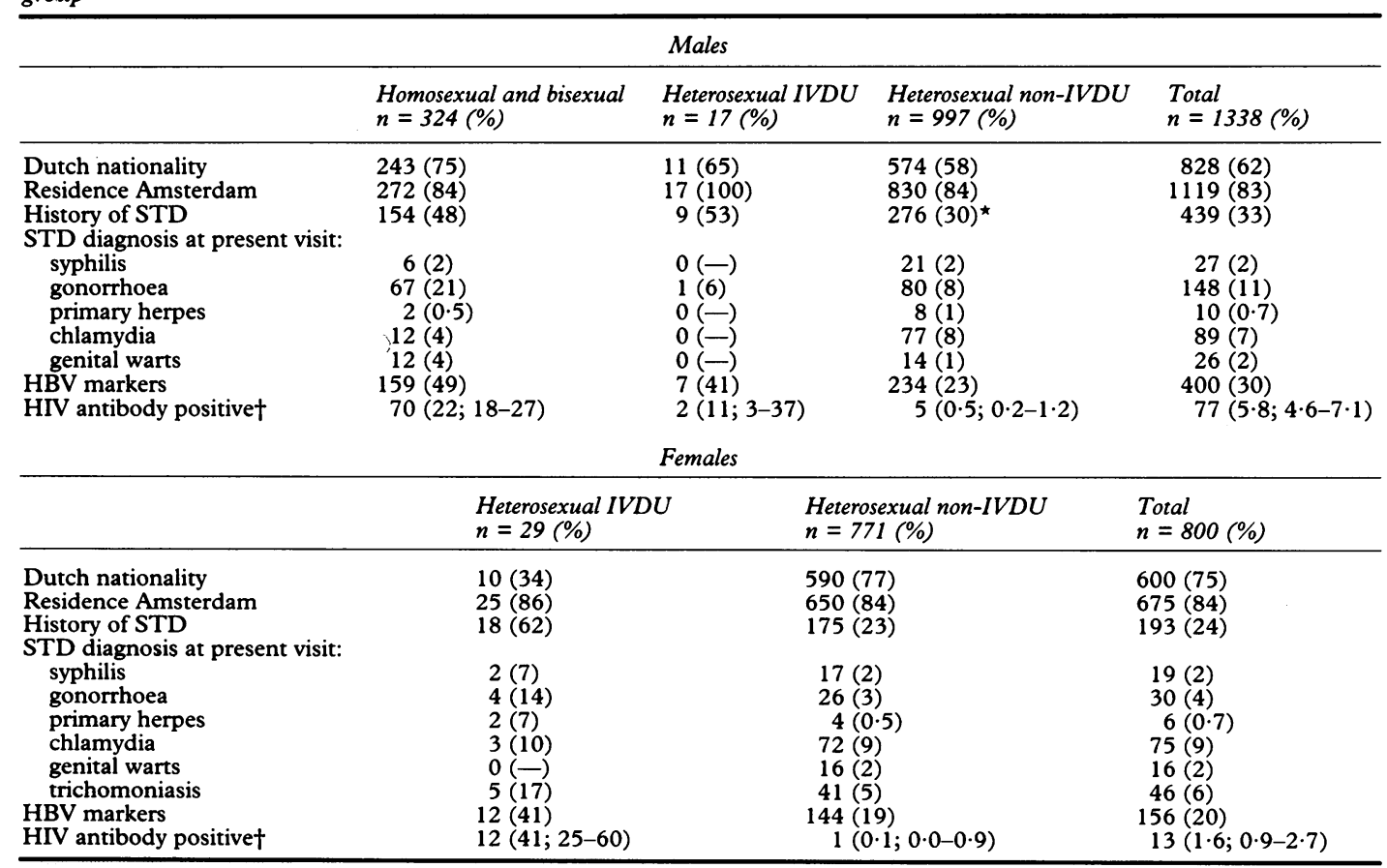

$t=$ between ( ): prevalence and $95 \%$ confidence interval.

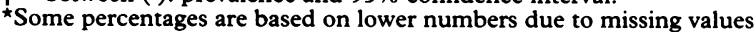


Table 3 Selected characteristics of six heterosexual, non-intravenous drug using HIV infected attenders of an STD clinic

\begin{tabular}{lllllll}
\hline & 1 & 2 & 3 & 4 & 5 & 6 \\
\hline Gender & Male & Male & Male & Male & Male & Female \\
Country of birth & Pakistan & Netherlands & Turkey & Turkey & Ghana & Netherlands \\
Residence Amsterdam & Yes & Yes & Yes & Yes & Yes & Yes \\
Tested previously & No & No & No & No & No & Yes (neg) \\
History of STD $\dagger$ & No & No & Syphilis & No & Syphilis & No \\
Number of heterosexual partners & 1 & $2-4$ & $>10$ & $>10$ & $2-4$ & $5-10$ \\
Commercial sex partners & No & No & Yes & Yes & No & Yes \\
Sexual partner HIV infected $\dagger$ & No & No & No & No & No & No \\
STD diagnosis at present visit & Chlamydia & None & Gonorrhoea & Syphilis & Syphilis & None \\
Transfusion recipient $\dagger$ & No & No & No & No & No & No \\
\hline
\end{tabular}

$\star=$ in the preceding six months.

$\dagger=$ in the last ten years.

Table 4 Number of sexual partners and STD diagnoses in 90 seropositive STD clinic attenders, by gender and sexual orientation

\begin{tabular}{|c|c|c|c|c|c|}
\hline & & \multicolumn{2}{|l|}{ Men } & \multirow{2}{*}{$\begin{array}{l}\text { Women } \\
n=13 t\end{array}$} & \multirow{2}{*}{$\begin{array}{l}\text { Total } \\
n=90\end{array}$} \\
\hline & & $\begin{array}{l}\text { Exclusive homosexual } \\
n=62\end{array}$ & $\begin{array}{l}\text { Hetero/bisexual } \\
n=15 t\end{array}$ & & \\
\hline \multicolumn{2}{|c|}{$\begin{aligned} \text { Number of sexual partners in last six months: } & 1 \\
& 2-4 \\
5-10 & >10\end{aligned}$} & $\begin{array}{r}10(16 \%) \\
26(42 \%) \\
6(10 \%) \\
19(31 \%)\end{array}$ & $\begin{array}{l}4^{\star}(26 \%) \\
7^{\star}(47 \%) \\
1^{\star}(6 \%) \\
3^{\star}(20 \%)\end{array}$ & $\begin{array}{l}4(31 \%) \\
2(15 \%) \\
2(15 \%) \\
5(38 \%)\end{array}$ & $\begin{array}{r}18(20 \%) \\
35(39 \%) \\
9(10 \%) \\
27(30 \%)\end{array}$ \\
\hline \multirow{4}{*}{\multicolumn{2}{|c|}{$\begin{aligned} & \text { Number diagnosed with STD: } \text { syphilis } \\
& \text { gonorrhoea } \\
& \text { primary herpes } \\
& \text { chlamydia infection } \\
& \text { genital warts } \\
& \text { trichomoniasis }\end{aligned}$}} & $\begin{array}{r}3 \\
15\end{array}$ & $\begin{array}{l}2 \\
4\end{array}$ & $\begin{array}{l}1 \\
2\end{array}$ & $\begin{array}{r}6 \\
21\end{array}$ \\
\hline & & 2 & - & 2 & 4 \\
\hline & & 2 & 1 & 2 & 5 \\
\hline & & 1 & - & -2 & 1 \\
\hline \multicolumn{2}{|l|}{$\begin{array}{l}\text { Number previously tested } \\
- \text { of whom known HIV positive }\end{array}$} & $\begin{array}{l}\overline{30} \\
25(83 \%)\end{array}$ & $\begin{array}{l}5 \\
4(80 \%)\end{array}$ & $\begin{array}{l}13 \\
9(69 \%)\end{array}$ & $\begin{array}{l}48 \\
48(79 \%)\end{array}$ \\
\hline
\end{tabular}

$\star=$ for hetero/bisexual men only heterosexual partners are shown.

$\dagger=$ including IVDU.

and four women apparently had seroconverted since their last test. All six male seroconverters were homosexual or bisexual men; three of four female seroconverters were IVDU while one woman apparently contracted an HIV infection through heterosexual contact.

STD Prevalence, number of sexual partners and the risk of further sexual transmission of HIV among seropositive participants

To assess the potential risk of further sexual transmission of HIV, we estimated the total number of homosexual and heterosexual partners reported by HIV seropositive participants and determined the number of STDs diagnosed at the present clinic-visit in this group (table 4 ). The minimum and maximum number of reported sexual partners was estimated by adding up the products of the number of respondents within each category and the minimum and maximum of this category respectively.

Among $62 \mathrm{HIV}$ infected men who were exclusively homosexual, $51(84 \%)$ reported more than one sexual partner in the six months preceding the study; these $62 \mathrm{HIV}$ infected men together had sexual contact with between 280 and 350 partners in the last 6 months. In 21 of the 62 men one or more STD was diagnosed: three cases of syphilis, 15 cases of gonorrhoea, two cases of primary genital herpes, two cases of genital chlamydia and one case with a first episode of genital warts.

Recent heterosexual partners were reported by 28 of 90 HIV infected participants: 15 men and 13 women. These $28 \mathrm{HIV}$ seropositive participants include bisexual men and IVDU: together they reported between 120 and 150 heterosexual partners in the six months preceding the study, while three men and eight women in this group reported commercial sexual partners in this period. The inconsistent use of condoms is illustrated by the number of STD diagnosed in 13 of these 28 participants: three cases of syphilisamong them two cases of early syphilis-, six cases of gonorrhoea, two cases of primary genital herpes, three cases of genital chlamydia and two cases of trichomoniasis. Of these 13 STD patients, none of seven men had been tested previously; four of the six women already knew they were seropositive. Among exclusively homosexual men as well as among heterosexually active participants the prevalence of STD and the number of reported sexual partners did not differ significantly between seropositive participants who already knew they were HIV infected from previous HIV tests and those who had tested negative or were never tested previously.

Among the six participants who are assumed to be HIV infected through heterosexual contact, two of the five men reported commercial sexual contacts. The only woman infected through heterosexual contact has been active as a prostitute and reported 5-10 clients in the six months preceding the study. In four of these six heterosexually infected participants two cases of syphilis-including one case of early syphilis-, one case of gonorrhoea and one case of chlamydia were diagnosed (table 3 ). 


\section{Discussion}

The extent to which HIV is transmitted among heterosexually active individuals is crucial for the future course of the epidemic. The HIV prevalence was $4 \cdot 2 \%$ among clients attending the STD clinic in Amsterdam. Homosexual and bisexual men and IVDU accounted for $93 \%$ of HIV infected persons in this study. The six HIV infected participants who denied homosexual contacts and intravenous use of drugs are assumed to be infected through heterosexual contact, resulting in a $0.35 \%$ prevalence among non-IVDU heterosexuals.

However, to assess the potential risk of further heterosexual transmission of HIV, the HIV prevalence among participants who reported heterosexual contacts is of importance. The HIV prevalence among heterosexually active participants was $1.5 \%$. This group includes intravenous drug users and bisexual men and 28 of them were HIV seropositive. Together these 28 participants reported between 120 and 150 heterosexual partners in the six months preceding the study. The potential risk, however, of sexual transmission of HIV is not only dependent of the number of sexual partners, but also of the techniques practised, the use of condoms and of the serostatus of the partners. The results of this study are limited by the fact that information on these issues was not collected. It seems apparent however, that those attending the STD clinic do so because they have engaged in sexual contacts which has placed them at risk of contracting an STD. Consequently, sexual partners of seropositive clinic attenders may have been at risk of exposure to HIV. This risky sexual behaviour is reflected by the 16 cases of STDs in 13 of the 28 HIV infected heterosexually active participants and 23 cases of STDs in 21 of the 62 exclusively homosexual seropositive men. Among heterosexual men, 30\% (4/13) of HIV positive participants with a STD diagnosed at the present clinic visit already knew they were HIV infected. Studies have shown that the presence of STD, in particular ulcerative STD, may greatly enhance sexual transmission of HIV.7-13

In order to try to reduce the risk of sexual transmission of HIV, a change towards safer sexual behaviour is necessary. Some studies have shown that this change may be stimulated by knowledge of one's serostatus. ${ }^{14-17}$ Therefore, services offered by the STD clinic should include voluntary counselling and confidential HIV testing. Recent studies showed partner notification of HIV positive STD patients to be effective for tracing sexual partners who themselves were HIV infected or exposed to HIV without knowing so. ${ }^{18-20}$ Thus, in addition to voluntary counselling and testing, notification, in particular of heterosexual partners of seropositive clinic visitors may contribute to a risk reduction.

Members of certain minority groups who visit the clinic, specifically Turkish men and those originating from Surinam and the Dutch Antilles, have been shown to be at increased risk for STD. ${ }^{23}$ Because cultural values and language difficulties can be obstacles for participation in studies like this one, visitors belonging to these minority groups were approached by native tongue speaking ethnic interviewers. This resulted in complete participation of Turkish men and greatly improved participation rates for visitors from Surinam and the Dutch Antilles in the second 5-week study period.

Since participation is voluntary in this study, the results can be biased through selection of participants. ${ }^{21}$ Unlinked anonymous screening of blood samples taken for other purposes would be effective to avoid participation bias, but is less informative on HIV related risk behaviours than voluntary surveys. ${ }^{22}$ The design of our study allowed for linking of anonymous HIV test results to specific information on risk behaviour. Of patients attending the clinic, $3 \%$ rejected any participation in this study and of those who participated less than $7 \%$ refused to have their blood tested. Differences, between participants who were tested and who refused testing, concerned prior STD episodes, involvement in commercial sex and a history of intravenous use of drugs. Though these differences suggest that the results of this study may be slightly biased, the direction of this bias is not clear.

From the results of this study several conclusions can be drawn. First, the HIV prevalence among heterosexual clinic visitors who never injected drugs is low. However, the HIV prevalence among visitors who are heterosexually active, including IVDU and bisexual men, is approximately $1 \cdot 5 \%$. Both the reported total number of heterosexual partners and the number of STD diagnosed in these HIV infected heterosexually active clinic visitors suggest that this group may play an important role in further transmission of HIV through heterosexual contact. To help reduce the further spread of HIV through sexual contact, services offered by the clinic should not only include counselling and confidential HIV testing but also notification of partners (particularly heterosexual partners) of HIV infected clinic visitors. Finally, it is concluded that "linked" anonymous HIV prevalence studies like this one can be performed with a high participation rate for collecting information on risk behaviour for HIV infection. Over time serial performance of this type of prevalence measurement provides information that can help estimating the HIV incidence in the various transmission categories.

The authors thank JAPCM Kint, MA Spijkers, HM van Bijnen, J Walter, K Cinabulak and D Beneck for their efforts made to interview almost all the clients who attended the clinic during the study period. We clients who attended the clinic during the study period. We also thank the personnel of the clinic for sexually transmitted diseases of The Municipa Health Service in Amsterdam for flexibility and sense of coop-

This study was financially supported by the Dutch Foundation for Preventive Medicine (grant no 28-1525). 
1 Holmes $\mathrm{KK}$, Karon JM, Kreiss J. The increasing frequency of heterosexually acquired AIDS in the United States, 1983-88. Am $\mathcal{F}$ Public Health 1990;80:858-62.

2 Anonymous. AIDS surveillance in Europe, Quarterly report no. 32, December 1991, WHO Collaborating Centre on AIDS, Paris.

3 Anonymous. HIV prevalence estimates and AIDS case projections for the United States; Report based upon a workshop. MMWR 1990;39:RR1 6:7-9.

4 The European Study Group. Surveillance of HIV seroprevalence among STD patients in European countries. VII International Conference on AIDS, Florence, 1991, Abstract NC 3223.

5 Haastrecht HJA van, Hoek JAR van den, Coutinho RA. Evidence for a change in behaviour among heterosexuals in Amsterdam under the

6 Annual report of the clinic for sexually transmitted diseases, 1990. Department of Public Health and Environment, Municipal Health Service of Amsterdam, 1991. (in Dutch)

7 Holmberg SD, Stewart JA, Gerber AR et al. Prior herpes simplex virus type 2 infection as a risk factor for HIV infection. FAMA 1988;259:1048-50.

8 Simmson JN, Cameron DW, Gakinya MN et al. Human immunodeficiency virus infection among men with sexually transmitted diseases: experience from a centre in Africa. $N$ Engl F Med 1988;319:274-8.

9 Stamm WE, Handsfield HH, Rompalo AM, et al. The association between genital ulcer disease and acquisition of HIV infection in homosexual men. $\mathscr{f} A M A 1988$; 260:1429-33.

10 Plummer FA, Simonsen JN, Kreiss JK, et al. Cofactors in male-female sexual transmission of Human Immunomale-female sexual transmission of Human Immuno-

11 Perre van de $\mathrm{Ph}$, de Clerca A, Cogniaux-Leclerc J, et al. Detection of HIV pl7 antigen in lymphocytes but not in epithelial cells from cervicovaginal secretion of wome seropositive for HIV: implications for heterosexual transmission of the virus. Genitourin Med 1988;64:30-3

12 Laga M, Nzila N, Manoka AT, et al. Non ulcerative sexually transmitted diseases as a risk factor for HIV infection. VI International Conference on AIDS, San Francisco, California, USA 1990, abstract Th.C.97.

13 Piot $P$, Laga $M$. Genital ulcers, other sexually transmitted diseases, and the sexual transmission of HIV. $B M \mathcal{F}$ 1989;298:623-4

14 Griensven van GJP, Vroome de EMM, Tielman RAP et al. Impact of HIV antibody testing on changes in sexual behaviour among homosexual men in the Netherlands. Am $\mathcal{F}$ Public Health 1988;78:1575-7.

15 Hoek van den JAR, Haastrecht van HJA, Coutinho RA. Little change in sex behaviour in injecting drug users in Amsterdam. $\mathcal{f}$ AIDS 1992;5:518-22.

16 Hoek van den JAR, Haastrecht van HJA, Cuotinho RA. Heterosexual behaviour of intravenous drug users in Amsterdam: implications for the AIDS epidemic AIDS 1990;4:449-53.

17 Higgins DL, Galavotti C, O'Reilly KR, et al. Evidence for the effects of HIV antibody counselling and testing on risk behaviours. $¥ A M A$ 1991; 266:2419-29.

18 W/ykf RF, Jone JL, Langshore ST et al Notification of the sex and needle-sharing partners of individuals with human immunodeficiency virus in rural South Carolina: 30 months experience. Sexually Transmitted Diseases 1991;18:217-22.

19 Giesecke J, Ramstedt K, Granath F, et al. Efficacy of partner notification for HIV infection. Lancet 1991;338: 1096-100.

20 Landis SE, Schoenbach BJ, Weber DJ et al. Results of a randomized trial of partner notification in cases of HIV infection in North Carolina. N Engl f Med 1992;326: 101-6.

21 Hull HF, Bettinger CJ, Gallaher MM, et al. Comparison of HIV antibody prevalence in patients consenting to and declining HIV antibody testing in an STD clinic. and declining $1988 ; 260: 935-8$.

22 Unlinked anonymous screening for the public health surveillance of HIV infection; proposed international veillance of HIV infection;

23 Hooykaas C, Velde van der FM, Linden van der MMD, et al. The importance of ethnicity as a risk factor for STDs and sexual behaviour among heterosexuals. Genitourin Med 1991;67:378-83. 